\title{
On S. Mazur's problems 8 and 88 from the Scottish Book
}

\author{
by \\ V. V. PELler (East Lansing, MI)
}

\begin{abstract}
The paper discusses Problems 8 and 88 posed by Stanisław Mazur in the Scottish Book. It turns out that negative solutions to both problems are immediate consequences of the results of Peller [J. Operator Theory 7 (1982)]. We discuss here some quantitative aspects of Problems 8 and 88 and give answers to open problems discussed in a recent paper of Pełczyński and Sukochev in connection with Problem 88.
\end{abstract}

1. Introduction. We are going to discuss Problems 8 and 88 posed by Stanisław Mazur in the Scottish Book [SB]. Problem 88 asks whether a Hankel matrix in the injective tensor product $\ell^{1} \check{\otimes} \ell^{1}$ of two spaces $\ell^{1}$ must have finite sum of the moduli of its matrix entries. Problem 8 asks whether for an arbitrary sequence $\left\{z_{n}\right\}_{n \geq 0}$ in the space $c$ of converging sequences there exist sequences $\left\{x_{n}\right\}_{n \geq 0}$ and $\left\{y_{n}\right\}_{n \geq 0}$ in $c$ such that

$$
z_{n}=\frac{1}{n+1} \sum_{k=0}^{n} x_{k} y_{n-k}, \quad n \geq 0 .
$$

We give precise statements of the problems and all necessary definitions later.

It is known that both problems have negative solutions. Independently, solutions were obtained by Kwapień and Pełczyński [KP] and Eggermont and Leung [EL]. In a recent paper by Pełczyński and Sukochev [PS] in Section 6 certain quantitative results related to negative solutions of Problems 8 and 88 are obtained and certain open problems are raised.

It turns out, however, that the results of Section 5 of my earlier paper [P1] immediately imply negative solutions to Problems 8 and 88. Moreover, Section 5 of [P1] contains much stronger results. In particular, a complete description of the Hankel matrices $\left({ }^{1}\right)$ in the injective tensor product of two

2000 Mathematics Subject Classification: 47B35, 46M05, 47A07, 46E35.

Key words and phrases: Hankel matrix, injective tensor product, projective tensor product, Besov spaces, Fourier coefficients.

The author is partially supported by NSF grant DMS 0200712 .

$\left({ }^{1}\right)$ Note that Hankel matrices and Hankel operators play an important role in many areas of mathematics and applications; see [P2]. 
spaces $\ell^{1}$ is obtained in [P1] in terms of the Besov space $B_{\infty, 1}^{1}$. Unfortunately, I was not aware of Problems 8 and 88 when I was writing the paper [P1].

In Sections 3 and 4 of this paper we explain why the results of [P1] immediately imply negative solutions to Problems 8 and 88 and we give a solution to the problems raised in [PS].

In $\S 2$ we collect necessary information on tensor products and Besov spaces.

\section{Preliminaries}

2.1. Projective and injective tensor products. We define the projective tensor product $\ell^{\infty} \widehat{\otimes} \ell^{\infty}$ as the space of matrices $\left\{q_{j k}\right\}_{j, k \geq 0}$ of the form

$$
q_{j k}=\sum_{n \geq 0} a_{j}^{(n)} b_{k}^{(n)},
$$

where $a^{(n)}=\left\{a_{j}^{(n)}\right\}_{j \geq 0}$ and $b^{(n)}=\left\{b_{j}^{(n)}\right\}_{j \geq 0}$ are sequences in $\ell^{\infty}$ such that

$$
\sum_{n \geq 0}\left\|a^{(n)}\right\|_{\ell^{\infty}}\left\|b^{(n)}\right\|_{\ell^{\infty}}<\infty .
$$

The norm of the matrix $\left\{q_{j k}\right\}_{j, k \geq 0}$ in $\ell^{\infty} \widehat{\otimes} \ell^{\infty}$ is defined as the infimum of the left-hand side of (2.2) over all sequences $a^{(n)}=\left\{a_{j}^{(n)}\right\}_{j \geq 0}$ and $b^{(n)}=$ $\left\{b_{j}^{(n)}\right\}_{j \geq 0}$ satisfying $(2.1)$.

Similarly, one can define the projective tensor products $c \widehat{\otimes} c$ and $c_{0} \widehat{\otimes} c_{0}$, where $c$ is the subspace of $\ell^{\infty}$ that consists of the converging sequences and $c_{0}$ is the subspace of $c$ that consists of the sequences with zero limit.

We consider the space $V^{2}$ that is a kind of a "weak completion" of $\ell^{\infty} \widehat{\otimes} \ell^{\infty} \cdot V^{2}$ consists of the matrices $Q=\left\{q_{j k}\right\}_{j, k \geq 0}$ for which

$$
\sup _{n>0}\left\|P_{n} Q\right\|_{\ell^{\infty} \hat{\otimes} \ell^{\infty}}<\infty,
$$

where the projections $P_{n}$ are defined by

$$
\left(P_{n} Q\right)_{j k}= \begin{cases}q_{j k} & \text { if } j, k \leq n \\ 0 & \text { otherwise. }\end{cases}
$$

Note that $c \widehat{\otimes} c \subset \ell^{\infty} \widehat{\otimes} \ell^{\infty} \subset V^{2}$.

The injective tensor product $\ell^{1} \ddot{\otimes} \ell^{1}$ of two spaces $\ell^{1}$ is, by definition, the space of matrices $Q=\left\{q_{j k}\right\}_{j, k \geq 0}$ such that

$$
\|Q\|_{\ell^{1} \dot{\otimes} \ell^{1}}=\sup \left|\sum_{j, k=0}^{N} q_{j k} x_{j} y_{k}\right|<\infty,
$$

where the supremum is taken over all sequences $\left\{x_{j}\right\}_{j \geq 0}$ and $\left\{y_{k}\right\}_{k \geq 0}$ in the unit ball of $\ell^{\infty}$ and over all positive integers $N$. The space $\ell^{1} \check{\otimes} \ell^{1}$ can be 
naturally identified with the space of bounded linear operators from $c_{0}$ to $\ell^{1}$ (note that every bounded operator from $c_{0}$ to $\ell^{1}$ is compact).

2.2. Besov spaces. In this paper we consider only Besov spaces of functions analytic in the unit disk $\mathbb{D}$. Besov spaces $B_{p, q}^{s}$ admit many equivalent descriptions. We give a definition in terms of dyadic Fourier expansions. We define the polynomials $W_{n}, n \geq 0$, as follows. If $n \geq 1$, then $\widehat{W}_{n}\left(2^{n}\right)=1$, $\widehat{W}_{n}(k)=0$ for $k \notin\left(2^{n-1}, 2^{n+1}\right)$, and $\widehat{W}_{n}$ is a linear function on $\left[2^{n-1}, 2^{n}\right]$ and on $\left[2^{n}, 2^{n+1}\right]$. We put $W_{0}(z)=1+z$. It is easy to see that

$$
\left\|W_{n}\right\|_{L^{1}} \leq 3 / 2, \quad n \geq 0
$$

and

$$
f=\sum_{n \geq 0} f * W_{n}
$$

for an arbitrary analytic function $f$ in $\mathbb{D}$.

For $1 \leq p, q \leq \infty$, and $s \in \mathbb{R}$, we define the Besov space $B_{p, q}^{s}$ as the space of analytic functions in $\mathbb{D}$ satisfying

$$
f \in B_{p, q}^{s} \Leftrightarrow\left\{2^{n s}\left\|f * W_{n}\right\|_{L^{p}}\right\}_{n \geq 0} \in \ell^{q} .
$$

If $q=\infty$, the space $B_{p, q}^{s}$ is nonseparable. We denote by $b_{p, \infty}^{s}$ the closure of the set of polynomials in $B_{p, \infty}^{s}$. It is easy to verify that

$$
f \in b_{p, \infty}^{s} \Leftrightarrow\left\{2^{n s}\left\|f * W_{n}\right\|_{L^{p}}\right\}_{n \geq 0} \in c_{0} .
$$

Besov spaces admit many other descriptions (see [Pe] and [P2]).

3. Problem 8. To state Mazur's Problem 8 of the Scottish Book [SB], consider the bilinear form $\mathcal{B}$ on $c \times c$ defined by

$$
\mathcal{B}\left(\left\{x_{n}\right\}_{n \geq 0},\left\{y_{n}\right\}_{n \geq 0}\right)=\left\{z_{n}\right\}_{n \geq 0},
$$

where

$$
z_{n}=\frac{1}{n+1} \sum_{k=0}^{n} x_{k} y_{n-k}, \quad n \geq 0 .
$$

It is easy to see that $B$ maps $c \times c$ into $c$. Stanisław Mazur asked in Problem 8 whether $\mathcal{B}$ maps $c \times c$ onto $c$.

As mentioned in the Introduction, a negative solution to Problem 8 follows immediately from the results of $\S 5$ of [P1]. To state Theorem 5.1 of [P1], we define an operator $\mathcal{A}$ on the space of matrices. Let $Q=\left\{q_{j k}\right\}_{j, k \geq 0}$. Then $\mathcal{A} Q$ is the sequence defined by

$$
\mathcal{A} Q=\left\{z_{n}\right\}_{n \geq 0}, \quad \text { where } \quad z_{n}=\frac{1}{n+1} \sum_{j+k=n} q_{j k} .
$$

Theorem (5.1 of [P1]). $\mathcal{A}$ maps the space $V^{2}$ onto the space of Fourier coefficients of the Besov space $B_{1, \infty}^{0}$. 
Recall that the space $V^{2}$ is defined in Section 2. In particular, it follows from Theorem 5.1 of [P1] that

$$
\mathcal{A}(c \widehat{\otimes} c) \subset \mathcal{A}\left(\ell^{\infty} \widehat{\otimes} \ell^{\infty}\right) \subset \mathcal{A}\left(V^{2}\right) \subset\left\{\{\widehat{f}(n)\}_{n \geq 0}: f \in B_{1, \infty}^{0}\right\},
$$

and so

$$
\mathcal{B}(c \times c) \subset\left\{\{\widehat{f}(n)\}_{n \geq 0}: f \in B_{1, \infty}^{0}\right\} .
$$

It is easy to see that

$$
c \not \subset\left\{\{\widehat{f}(n)\}_{n \geq 0}: f \in B_{1, \infty}^{0}\right\} .
$$

Indeed, if $f \in B_{1, \infty}^{0}$, then it follows immediately from (2.3) and from [R, $\S 8.6]$ that

$$
\sup _{n \geq 0} \sum_{k=0}^{n}\left|\widehat{f}\left(2^{n}+2^{k}\right)\right|^{2}<\infty .
$$

This gives a negative solution to Problem 8 .

In fact, Theorem 5.1 of $[\mathrm{P} 1]$ allows one to describe $\mathcal{A}(c \widehat{\otimes} c)$. First, let us observe that Theorem 5.1 of [P1] easily implies the following description of $\mathcal{A}\left(c_{0} \widehat{\otimes} c_{0}\right)$.

THEOREM 3.1.

$$
\mathcal{A}\left(c_{0} \widehat{\otimes} c_{0}\right)=\left\{\{\widehat{f}(n)\}_{n \geq 0}: f \in b_{1, \infty}^{0}\right\} .
$$

Recall that $b_{1, \infty}^{0}$ is the closure of the polynomials in $B_{1, \infty}^{0}$ (see $\S 2$ ). Theorem 3.1, in turn, easily implies the following description of $\mathcal{A}(c \widehat{\otimes} c)$.

THEOREM 3.2 .

$$
\mathcal{A}(c \widehat{\otimes} c)=\left\{\{\widehat{f}(n)+d\}_{n \geq 0}: f \in b_{1, \infty}^{0}, d \in \mathbb{C}\right\} .
$$

4. Problem 88. Recall that in Problem 88 of [SB], S. Mazur asked whether a Hankel matrix $\left\{\gamma_{j+k}\right\}_{j, k \geq 0}$ in the injective tensor product $\ell^{1} \check{\otimes} \ell^{1}$ must satisfy the condition

$$
\sum_{k=0}^{\infty}(1+k)\left|\gamma_{k}\right|<\infty,
$$

i.e., whether the sum of the moduli of the matrix entries must be finite.

As mentioned in the Introduction, a negative solution to Problem 88 follows immediately from the results of $\S 5$ of [P1]. A complete description of Hankel matrices in $\ell^{1} \check{\otimes} \ell^{1}$ is given by Theorem 5.2 of [P1]:

Theorem (5.2 of [P1]). A Hankel matrix $\left\{\gamma_{j+k}\right\}_{j, k \geq 0}$ belongs to $\ell^{1} \check{\otimes} \ell^{1}$ if and only if the function $f$ defined by

$$
f(z)=\sum_{n \geq 0} \gamma_{n} z^{n}
$$

belongs to the Besov class $B_{\infty, 1}^{1}$. 
Let us obtain the best possible estimate on the moduli of the matrix entries of Hankel matrices in $\ell^{1} \check{\otimes} \ell^{1}$.

Since $\left\|f * W_{n}\right\|_{L^{2}} \leq\|f\|_{L^{2}}\left\|W_{n}\right\|_{L^{1}} \leq \frac{3}{2}\|f\|_{L^{2}}$, it follows easily from (2.3) that if $f \in B_{\infty, 1}^{1}$, then

$$
\sum_{n=0}^{\infty} 2^{n}\left(\sum_{k=2^{n}}^{2^{n+1}-1}|\widehat{f}(k)|^{2}\right)^{1 / 2}<\infty
$$

Let us show that this is the best possible estimate for the moduli of the Fourier coefficients of functions in $B_{\infty, 1}^{1}$. To show this, we are going to use a version of the de Leeuw-Katznelson-Kahane theorem. It was proved in [dLKK] that if $\left\{\beta_{n}\right\}_{n \in \mathbb{Z}}$ is a sequence of nonnegative numbers in $\ell^{2}(\mathbb{Z})$, then there exists a continuous function $f$ on $\mathbb{T}$ such that

$$
|\widehat{f}(n)| \geq \beta_{n}, \quad n \in \mathbb{Z} .
$$

We refer the reader to [K1], [K2], and [N] for refinements of the de LeeuwKatznelson-Kahane theorem and different proofs. We need the following version of the de Leeuw-Katznelson-Kahane theorem:

LEMMA 4.1. There is a positive number $K$ such that for arbitrary nonnegative numbers $\beta_{0}, \beta_{1}, \ldots, \beta_{m}$, there exists a polynomial $f$ of degree $m$ such that

$$
|\widehat{f}(j)| \geq \beta_{j}, \quad 0 \leq j \leq n, \quad \text { and } \quad\|f\|_{L^{\infty}} \leq K\left(\sum_{j=0}^{n} \beta_{j}^{2}\right)^{1 / 2} .
$$

Lemma 4.1 follows easily from the results of [K2].

THEOREM 4.2. Let $\left\{\alpha_{k}\right\}_{k \geq 0}$ be a sequence of nonnegative numbers such that

$$
\sum_{n=0}^{\infty} 2^{n}\left(\sum_{k=2^{n}}^{2^{n+1}-1} \alpha_{k}^{2}\right)^{1 / 2}<\infty
$$

Then there exists a function $\varphi$ in the space $B_{\infty, 1}^{1}$ such that $|\widehat{\varphi}(k)| \geq \alpha_{k}$ for $k \geq 0$.

Proof. By Lemma 4.1, there exists $K>0$ and a sequence of polynomials $f_{n}, n \geq 0$, such that

$$
\begin{gathered}
f_{0}(z)=\widehat{f}_{0}(0)+\widehat{f}_{0}(1) z, \quad f_{n}(z)=\sum_{k=2^{n}}^{2^{n+1}-1} \widehat{f}_{n}(k) z^{k} \quad \text { for } n \geq 1, \\
\left|\widehat{f}_{0}(k)\right| \geq \alpha_{k} \quad \text { for } k=0,1, \quad\left|\widehat{f}_{n}(k)\right| \geq \alpha_{k} \quad \text { for } n \geq 1,2^{n} \leq k \leq 2^{n+1}-1,
\end{gathered}
$$


and

$$
\left\|f_{0}\right\|_{L^{\infty}} \leq K\left(\alpha_{0}^{2}+\alpha_{1}^{2}\right)^{1 / 2}, \quad\left\|f_{n}\right\|_{L^{\infty}} \leq K\left(\sum_{k=2^{n}}^{2^{n+1}-1} \alpha_{k}^{2}\right)^{1 / 2} \quad \text { for } n \geq 1 .
$$

We can now define the function $\varphi$ by

$$
\varphi=\sum_{n \geq 0} f_{n} .
$$

Obviously, $|\widehat{\varphi}(k)| \geq \alpha_{k}$ for $k \geq 0$. Let us show that $\varphi \in B_{\infty, 1}^{1}$. We have

$$
\begin{aligned}
\sum_{n \geq 1} 2^{n}\left\|\varphi * W_{n}\right\|_{L^{\infty}} & =\sum_{n \geq 1} 2^{n}\left\|\left(f_{n-1}+f_{n}+f_{n+1}\right) * W_{n}\right\|_{L^{\infty}} \\
& \leq \sum_{n \geq 1} 2^{n}\left\|\left(f_{n-1}+f_{n}+f_{n+1}\right)\right\|_{L^{\infty}}\left\|W_{n}\right\|_{L^{1}} \\
& \leq 3 \sum_{n \geq 1} 2^{n}\left\|f_{n}\right\|_{L^{\infty}}\left\|W_{n}\right\|_{L^{1}} \leq 3 \cdot \frac{3}{2} \sum_{n \geq 1} 2^{n}\left\|f_{n}\right\|_{L^{\infty}} \\
& \leq \frac{9}{2} K \sum_{n=0}^{\infty} 2^{n}\left(\sum_{k=2^{n}}^{2^{n+1}-1} \alpha_{k}^{2}\right)^{1 / 2}<\infty .
\end{aligned}
$$

In [PS] the following problem was considered. Let $\Psi$ be the function on $(0, \infty)$ defined by

$$
\Psi(t)= \begin{cases}\frac{3}{2} t-1, & 0<t \leq 2 \\ t, & t>2\end{cases}
$$

Let $\left\{\gamma_{j+k}\right\}_{j, k \geq 0}$ be a Hankel matrix. The following result was proved in [PS] (Theorem 6.7):

(i) if $\beta<\Psi(t)$, then

$$
\sum_{k \geq 0}\left|\gamma_{k}\right|^{t}(1+k)^{\beta}<\infty \quad \text { whenever }\left\{\gamma_{j+k}\right\}_{j, k \geq 0} \in \ell^{1} \check{\otimes} \ell^{1} ;
$$

(ii) if $\beta>\Psi(t)$, then

$$
\sum_{k \geq 0}\left|\gamma_{k}\right|^{t}(1+k)^{\beta}=\infty \quad \text { for some }\left\{\gamma_{j+k}\right\}_{j, k \geq 0} \in \ell^{1} \check{\otimes} \ell^{1} ;
$$

(iii) if $\beta=\Psi(t)$ and $4 / 3 \leq t<\infty$, then

$$
\sum_{k \geq 0}\left|\gamma_{k}\right|^{t}(1+k)^{\beta}<\infty \quad \text { whenever }\left\{\gamma_{j+k}\right\}_{j, k \geq 0} \in \ell^{1} \check{\otimes} \ell^{1} .
$$

In [PS] the problem is raised of finding whether

$$
\sum_{k \geq 0}\left|\gamma_{k}\right|^{t}(1+k)^{\Psi(t)}
$$

has to be finite for $t \in(0,4 / 3)$ whenever $\left\{\gamma_{j+k}\right\}_{j, k \geq 0} \in \ell^{1} \check{\otimes} \ell^{1}$. 
It is easy to deduce Theorem 6.7 of [PS] from (4.1) and the above Theorem 4.2. Moreover, using (4.1) and Theorem 4.2, we can solve the problem posed in [PS] and settle the case $t \in(0,4 / 3)$.

THEOREM 4.3. If $1 \leq t<4 / 3$, then

$$
\sum_{k \geq 0}\left|\gamma_{k}\right|^{t}(1+k)^{3 t / 2-1}<\infty \quad \text { whenever }\left\{\gamma_{j+k}\right\}_{j, k \geq 0} \in \ell^{1} \check{\otimes} \ell^{1} .
$$

If $0<t<1$, then

$$
\sum_{k \geq 0}\left|\gamma_{k}\right|^{t}(1+k)^{3 t / 2-1}=\infty \quad \text { for some }\left\{\gamma_{j+k}\right\}_{j, k \geq 0} \in \ell^{1} \check{\otimes} \ell^{1} .
$$

Proof. Suppose that $1 \leq t<2$. By Hölder's inequality, we have

$$
\begin{aligned}
\sum_{k \geq 1}\left|\gamma_{k}\right|^{t}(1+k)^{3 t / 2-1} & \leq \text { const } \sum_{n \geq 0} 2^{n(3 t / 2-1)} \sum_{k=2^{n}}^{2^{n+1}}\left|\gamma_{k}\right|^{t} \\
& \leq \text { const } \sum_{n \geq 0} 2^{3 n t / 2} 2^{-n}\left(\sum_{k=2^{n}}^{2^{n+1}}\left|\gamma_{k}\right|^{2}\right)^{t / 2} 2^{n(1-t / 2)} \\
& =\text { const } \sum_{n \geq 0} 2^{n t}\left(\sum_{k=2^{n}}^{2^{n+1}}\left|\gamma_{k}\right|^{2}\right)^{t / 2} .
\end{aligned}
$$

Since $t \geq 1$, the $\ell^{t}$ norm of a sequence does not exceed its $\ell^{1}$ norm, and so

$$
\sum_{n \geq 0} 2^{n t}\left(\sum_{k=2^{n}}^{2^{n+1}}\left|\gamma_{k}\right|^{2}\right)^{t / 2} \leq\left(\sum_{n \geq 0} 2^{n}\left(\sum_{k=2^{n}}^{2^{n+1}}\left|\gamma_{k}\right|^{2}\right)^{1 / 2}\right)^{t} .
$$

The result now follows from Theorem 5.2 of [P1] and (4.1).

Suppose now that $0<t<1$. It follows from Theorem 4.2 that it suffices to find a sequence $\left\{\alpha_{k}\right\}_{k \geq 0}$ of nonnegative numbers that satisfies (4.2) and

$$
\sum_{k \geq 0} \alpha_{k}^{t}(1+k)^{3 t / 2-1}=\infty .
$$

Let $\left\{\delta_{n}\right\}_{n \geq 0}$ be a sequence of positive numbers such that $\left\{2^{3 n / 2} \delta_{n}\right\}_{n \geq 0}$ $\in \ell^{1}$, but $\left\{2^{3 n / 2} \delta_{n}\right\}_{n \geq 0} \notin \ell^{t}$. Put

$$
\alpha_{0}=0, \quad \alpha_{k}=\delta_{n} \quad \text { if } 2^{n} \leq k \leq 2^{n+1}-1 .
$$

We have

$$
\sum_{n \geq 0} 2^{n}\left(\sum_{k=2^{n}}^{2^{n+1}-1} \alpha_{k}^{2}\right)^{1 / 2}=\sum_{n \geq 0} 2^{3 n / 2} \delta_{n}<\infty
$$


However,

$$
\begin{aligned}
\sum_{k \geq 0} \alpha_{k}^{t}(1+k)^{3 t / 2-1} & \geq \text { const } \sum_{n \geq 0} 2^{n(3 t / 2-1)} \sum_{k=2^{n}}^{2^{n+1}} \alpha_{k}^{t} \\
& =\text { const } \sum_{n \geq 0} 2^{n(3 t / 2-1)} 2^{n} \delta_{n}^{t}=\text { const } \sum_{n \geq 0} 2^{3 n t / 2} \delta_{n}^{t}=\infty
\end{aligned}
$$

\section{References}

[dLKK] K. de Leeuw, Y. Katznelson et J.-P. Kahane, Sur les coefficients de Fourier des fonctions continues, C. R. Acad. Sci. Paris Sér. A-B 285 (1977), A1001-A1003.

[EL] P. P. B. Eggermont and Y. J. Leung, On a factorization problem for convergent sequences and on Hankel forms in bounded sequences, Proc. Amer. Math. Soc. 96 (1986), 269-274.

[K1] S. V. Kislyakov, Fourier coefficients of boundary values of functions that are analytic in the disc and bidisc, Trudy Mat. Inst. Steklov. 155 (1981), 77-94 (in Russian); English transl.: Proc. Steklov Inst. Math. 155 (1983), 75-91.

[K2] - Fourier coefficients of continuous functions and a class of multipliers, Ann. Inst. Fourier (Grenoble) 38 (1988), 147-183.

[KP] S. Kwapień and A. Pełczyński, On two problems of S. Mazur from the Scottish $B o o k$, lecture at the Colloquium dedicated to the memory of Stanisław Mazur, Warsaw Univ., 1985 (unpublished).

[N] F. L. Nazarov, The Bang solution of the coefficient problem, Algebra i Analiz 9 (1997), 272-287 (in Russian); English transl.: St. Petersburg Math. J. 9 (1998), 407-419.

[Pe] J. Peetre, New Thoughts on Besov Spaces, Duke Univ. Press., Durham, NC, 1976.

[PS] A. Pełczyński and F. Sukochev, Some remarks on Toeplitz multipliers and Hankel matrices, Studia Math. 175 (2006), 175-204.

[P1] V. V. Peller, Estimates of functions of power bounded operators on Hilbert spaces, J. Operator Theory 7 (1982), 341-372.

[P2] -, Hankel Operators and Their Applications, Springer, New York, 2003.

[R] W. Rudin, Fourier Analysis on Groups, Wiley Classics Library, Wiley, New York, 1990.

[SB] The Scottish Book, R. D. Mauldin (ed.), Birkhäuser, Boston, MA, 1979.

Department of Mathematics

Michigan State University

East Lansing, MI 48824, U.S.A.

E-mail: peller@math.msu.edu

Received March 20, 2007

Revised version March 27, 2007 